\title{
Posterior Subtalar Dislocation
}

\author{
LAWREnCE CAMARDA, MD; UMBerto MartoranA, MD; MiChele D'ArienZO, MD
}

\section{ahstract}

Full article available online at OrthoSuperSite.com/view.asp?rlD=00000

A subtalar dislocation of the foot is an uncommon injury, and cases of posterior subtalar dislocation are even rarer. This injury is characterized by a simultaneous dislocation of talocalcaneal and talonavicular joints while tibiotalar and calcaneocuboid articulations remain intact. Subtalar dislocation is commonly accompanied by fractures of the malleoli, talus, or fifth metatarsal and by a rotational component of the subtalar joint. Subtalar dislocation can occur in any direction and produces significant deformity.

This article presents a case of pure posterior subtalar dislocation in an 80-year-old man who presented with pain and deformity in his left ankle following a motorcycle accident. Radiographs demonstrated dislocation of both the talonavicular and talocalcaneal joints. The calcaneus was displaced posteriorly with no medial or lateral displacement. No rotational component of the calcaneus was noted, suggesting the trauma was in pure hyperplantar flexion.

We hypothesize that pure hyperplantar flexion could lead to a progressive subtalar ligament weakening that may result in a complete ligament rupture if the plantar flexion force is prolonged. This could be observed in the presence of good bone quality and if the force is applied distally at the navicular bone.

Drs Camarda, Martorana, and D'Arienzo are from Clinica Ortopedica e Traumatologica, Università degli Studi di Palermo, Italy.

Drs Camarda, Martorana, and D'Arienzo have no relevant financial relationships to disclose.

Correspondence should be addressed to: Lawrence Camarda, MD, Dipartimento delle Chirurgie Speciali, Unità Operativa di Ortopedia e Traumatologia, Università degli Studi di Palermo, Via del Vespro, Palermo 90100, Italy.

DOI: $10.3928 / 01477447-20090527-25$

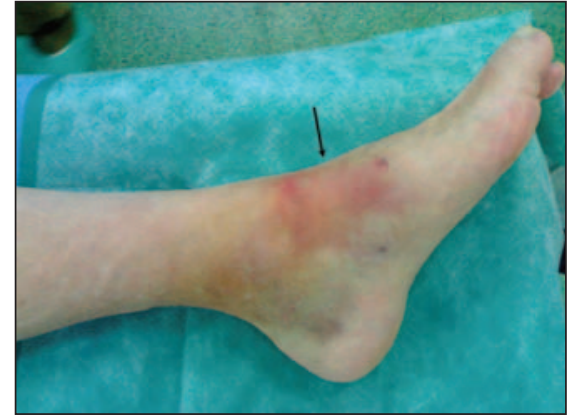

Figure: Clinical manifestation of posterior subtalar dislocation. The ankle is fixed in plantar flexion. The arrow shows the soft point that is delimited proximally by the articular surface of the talus head. 
$\mathrm{A}$ subtalar dislocation of the foot is an uncommon injury, and cases of posterior subtalar dislocation are even rarer. The injury is characterized by a simultaneous dislocation of talocalcaneal and talonavicular joints while tibiotalar and calcaneocuboid articulations remain intact. Subtalar dislocation is commonly accompanied by fractures of the malleoli, talus, or fifth metatarsal and by a rotational component of the subtalar joint. Subtalar dislocation can occur in any direction and produces significant deformity.

\section{CASE REPORT}

An 80-year-old man presented with pain and deformity in his left ankle following a motorcycle accident. Clinical examination revealed a slight swelling of the left ankle, which was fixed in plantar flexion. Anteriorly, a bony prominence was proximal to the navicular (Figure 1). The dorsalis pedis artery pulse was weak but present, and the sensation to light touch was intact. The patient was unable to actively move the ankle and reported severe pain when attempting passive movements. Radiographs demonstrated dislocation of both the talonavicular and talocalcaneal joints. The calcaneus was displaced posteriorly with no medial or lateral displacement (Figures 2-5).

Closed reduction under sedation was performed by longitudinal manual foot traction. With the left knee flexed and a countertraction applied to the leg, a firm digital pressure over the head of the talus was applied as the ankle was plantar flexed and then dorsiflexed. The reduction was associated with an audible clunk.

Post-reduction radiographs revealed a congruent reduction of the subtalar and talonavicular joints. The dorsalis pedus pulse returned to normal. The ankle was immobilized in a short leg cast, and the patient was discharged the day after the reduction. A computed tomography (CT) exam showed no osteochondral fracture.

The cast was removed after 3 weeks. The patient started partial weight bearing with crutches at 4 weeks, and progressive active and passive mobilization of the ankle was permitted. Full weight bearing was allowed at 8 weeks.
At 30-month follow-up, the patient reported no pain at rest or during ambulation. The ankle's active range of motion measured $15^{\circ}$ in dorsiflexion and $35^{\circ}$ in plantar flexion. Joint stress tests showed no instability compared with the controlateral ankle. Magnetic resonance imaging showed no evidence of avascular necrosis of the talus.

\section{DISCUSSION}

Subtalar dislocations are rare, accounting for $1 \%$ to $2 \%$ of all dislocations. $^{2}$ They are characterized by a concomitant loss of normal anatomical relations between the talus, calcaneus, and navicular. The first classification of subtalar dislocation is attributed to Broca $^{3}$ in 1853 . He described 3 different
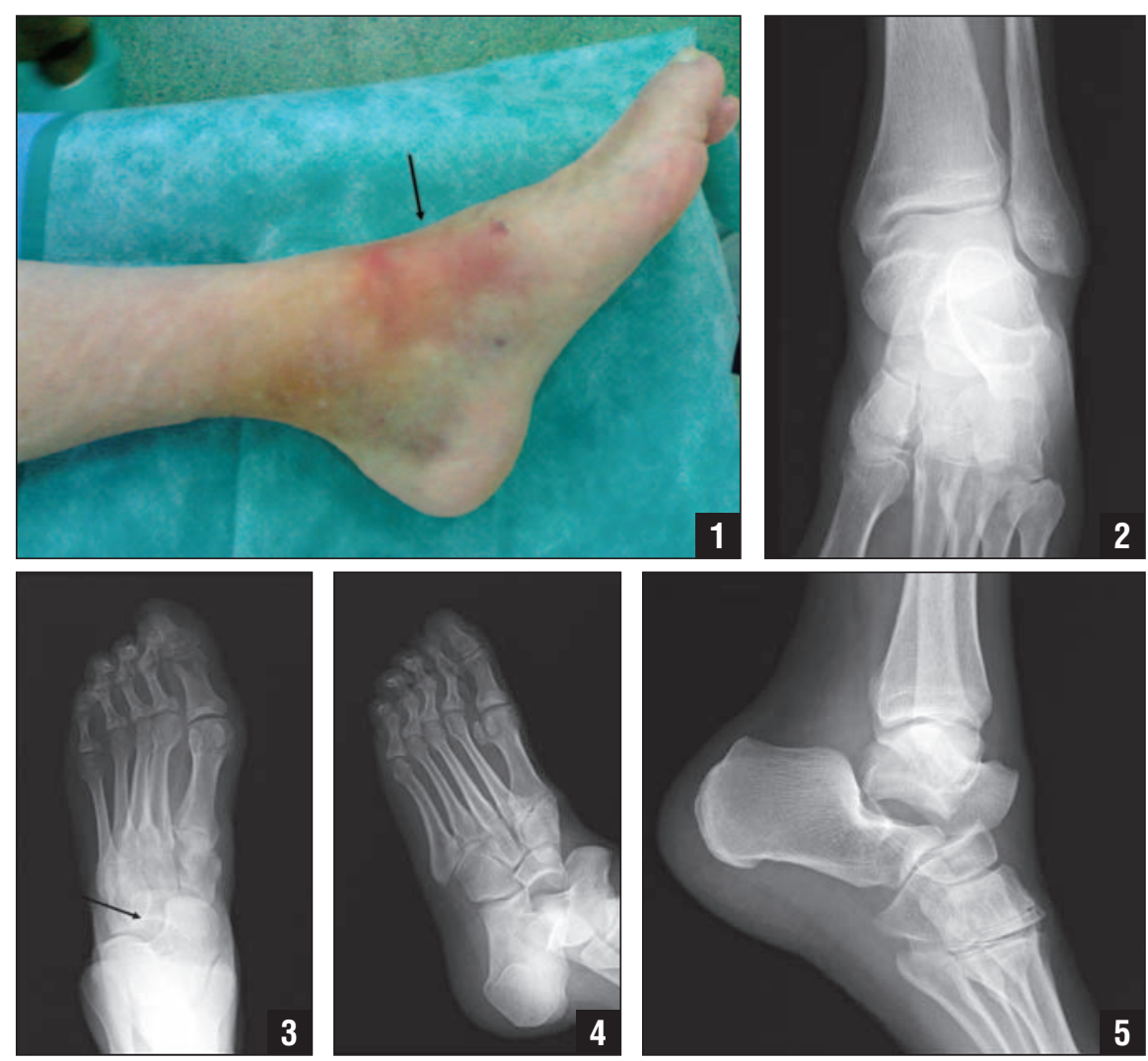

Figure 1: Clinical manifestation of posterior subtalar dislocation. The ankle is fixed in plantar flexion. The arrow shows the soft point that is delimited proximally by the articular surface of the talus head. Figure 2: AP radiograph showing no lateral or medial displacement of the foot. Figure 3: AP radiograph showing a normal angle value between the tibiotalar joint and the midfoot. The arrow shows the talonavicular joint dislocation. Figure 4: Lateral radiograph showing a true posterior subtalar dislocation. The posterior portion of the talus is in contact with the posterior subtalar facet of the calcaneus. The tibiotalar joint presents normal lateral angulations while the calcaneus and the midfoot is displaced posteriorly. Figure 5: Oblique radiograph showing a complete subtalar dislocation. 
Generally, subtalar dislocation occurs at an early age and in athletic young men as a result of a high-energy trauma such as a fall from a height or a motor vehicle accident. ${ }^{12}$ Subtalar dislocation is commonly accompanied by fractures of the malleoli, talus, or fifth metatarsal. Our patient was an active 80-year-old man with no comorbidities or previous fracture or joint dislocation.

Excessive plantar flexion is described as the main cause of posterior subtalar dislocation, whereas dorsiflexion leads to anterior subtalar dislocation. Inokuchi et $\mathrm{al}^{1}$ and Ogawa $^{12}$ suggest that the position of the foot is important to define the type of subtalar dislocation: supination or pronation of the foot at the time of injury leads to medial or lateral displacement. Usually subtalar dislocation occurs with an associate rotational component. Few reports of posterior subtalar dislocation have been described to date, and all of these describe medial or lateral displacement. ${ }^{13}$ In our case, there was no rotational component, suggesting that the trauma was in pure hyperplantar flexion; the foot was fixed in plantar flexion with no rotation of the calcaneus (Figure 2 ). We hypothesize that pure hyperplantar flexion could lead to a progressive subtalar ligament weakening that may result in a complete ligament rupture if the plantar flexion force is prolonged. This could be observed in the presence of good bone quality and if the force is applied distally at the navicular bone.

The diagnosis of posterior subtalar dislocation can be confirmed with lateral and anteroposterior radiographs. On lateral radiographs, the head of the talus is perched atop the navicular, and the posterior portion of the talus should be in contact with the posterior subtalar facet of the calcaneus (Figure 4). According to Inokuchi et al, ${ }^{1}$ the frontal view should show no significant medial-lateral displacement or rotation of the foot. Our patient presented these typical radiographic features (Figures 1, 2).

Immediate reduction under general or spinal anesthesia is recommended to avoid soft tissue complications and reduce the chances of avascular necrosis of the talus; however, medial and lateral dislocations may require an open reduction because of soft tissue interposition or a bony block. With posterior subtalar dislocation, reduction can be achieved with no difficulty by manual traction even if an avulsion fracture of the posterior malleolus occurs. ${ }^{14}$ The reduction should be performed with the left knee kept slightly flexed and a countertraction performed by the leg. At this point, ankle traction is applied, and a firm digital pressure over the head of the talus is performed from anterior to posterior, passing through plantar flexion to dorsiflexion. The reduction should be associated with an audible clunk. A radiograph should be performed to ensure the reduction of the dislocation and to exclude any iatrogenic fracture. Because of the risk of associated osteochondral fracture affecting the patient's clinical outcome, CT or MRI scans of the ankle and foot should be done post-reduction to evaluate the talus and sub-talus articular surface. In our patient, CT and MRI revealed no osteochondral fracture.

Good functional outcomes for closed posterior subtalar dislocation have been uniformly reported in the literature ${ }^{1,12-13}$; however, the prognosis may be poorer when posterior subtalar dislocation is associated with other lesions such as soft tissue injury, intra-articular fracture, extraarticular fracture, infection, osteonecrosis, and chronic sub-talus instability. ${ }^{11,15,16}$

Post-reduction immobilization in a nonweight-bearing cast is required for subtalar dislocation. The period of immobilization is controversial; Buckingam and LeFlore $^{17}$ and Zimmer and Johnson ${ }^{18}$ recommend immobilizing the ankle for $>4$ weeks, while DeLee and Curtis ${ }^{2}$ found that casting for 3 weeks leads to better outcomes. We prefer 3 weeks of immobilization followed by physical therapy to restore hindfoot range of motion, considering that prolonged casting can cause loss of function and subtalar joint arthrosis. No recurrent subtalar dislocation has been described in the literature to date, suggesting that residual subtalar joint laxity does not represent a risk for future recurrent subtalar dislocation.

\section{REFERENCES}

1. Inokuchi S, Hashimoto T, Usami N. Posterior subtalar dislocation. J Trauma. 1997 42(2):310-313.

2. DeLee JC, Curtis R. Subtalar dislocation of the foot. J Bone Joint Surg Am. 1982; 64(3):433-437.

3. Broca P. Mèmoire sur les luxations sous-astragaliennes. Mém Soc Chir. 1853; (3):241.

4. Malgaigne JF, Burger DC. Die Knochenbrüche und VerrenKungen. Stuttgart, Germany: Rieger; 1856; (11):820.

5. Saltzman C, Marsh JL. Hindfoot dislocations: when are they not benign? J Am Acad Orthop Surg. 1997; 5(4):192-198.

6. Smith H. Subastragalar dislocation. A report of seven cases. J Bone Joint Surg Am. 1937; (19):373-380.

7. Leitner B. Obstacles to reduction in subtalar dislocations. J Bone Joint Surg Am. 1954; 36(A:2):299-306

8. Grantham A. Medial subtalar dislocation: five cases with a common etiology. J Trauma. 1964; (4):845-849.

9. Larsen HW. Subastragalar dislocation (luxatio pedis sub talo): a follow-up report of eight cases. Acta Chir Scand. 1957; 113(5):380-392.

10. Dunn AW. Peritalar dislocation. Orthop Clin North Am. 1974; 5(1):7-18.

11. Edmunds I, Elliott D, Nade S. Open subtalar dislocation. Aust N Z J Surg. 1991; 61(9):681-686.

12. Ogawa K. Subtalar dislocation of the foot. Foot. 1996; (6):168-174

13. Krishnan KM, Sinha AK. True posterior dislocation of subtalar joint: a case report. J Foot Ankle Surg. 2003; 42(6):363-365.

14. Rivera F, Bertone C, Crainz E, Maniscalco P Filisio M. Peritalar dislocation: three case reports and literature review. Journal of Orthopaedics and Traumatology. 2003; (4):39-44.

15. de Palma L, Santucci A, Marinelli M, Borgogno E, Catalani A. Clinical outcome of closed isolated subtalar dislocations. Arch Orthop Trauma Surg. 2008; 128(6):593-598.

16. Freund KG. Subtalar dislocations: a review of the literature. J Foot Surg. 1989 28(5):429-432.

17. Buckingham WW Jr, LeFlore I. Subtalar dislocation of the foot. J Trauma. 1973; 13(9):753-765.

18. Zimmer TJ, Johnson KA. Subtalar dislocations. Clin Orthop Relat Res. 1989; (238):190-194. 